\title{
An Unsupervised Method for Building Sentence Simplification Corpora in Multiple Languages
}

\author{
Xinyu Lu * Jipeng Qiang ${ }^{* \dagger}$ Yun Li Yunhao Yuan Yi Zhu \\ Department of Computer Science, Yangzhou University, Jiangsu, China \\ \{181303216, jpqiang, liyun, yhyuan, zhuyi\}@yzu.edu.cn
}

\begin{abstract}
The availability of parallel sentence simplification (SS) is scarce for neural SS modelings. We propose an unsupervised method to build SS corpora from large-scale bilingual translation corpora, alleviating the need for SS supervised corpora. Our method is motivated by the following two findings: neural machine translation model usually tends to generate more high-frequency tokens and the difference of text complexity levels exists between the source and target language of a translation corpus. By taking the pair of the source sentences of translation corpus and the translations of their references in a bridge language, we can construct large-scale pseudo parallel SS data. Then, we keep these sentence pairs with a higher complexity difference as SS sentence pairs. The building SS corpora with an unsupervised approach can satisfy the expectations that the aligned sentences preserve the same meanings and have difference in text complexity levels. Experimental results show that SS methods trained by our corpora achieve the state-of-the-art results and significantly outperform the results on English benchmark WikiLarge.
\end{abstract}

\section{Introduction}

The task of sentence simplification (SS) is to rephrase a sentence into a form that is easier to read and understand while conveying the same meaning (Chandrasekar et al., 1996). SS is first used as a preprocessing task of machine translation, and then is used to increase accessibility for those with cognitive disabilities such as aphasia (Carroll et al., 1998), dyslexia (Rello et al., 2013), and autism (Evans et al., 2014).

Most popular methods (Wubben et al., 2012a; Xu et al., 2016; Zhang and Lapata, 2017; Nisioi et al., 2017; Martin et al., 2020a) have addressed

\footnotetext{
*Equal contribution.

${ }^{\dagger}$ Corresponding author.
}

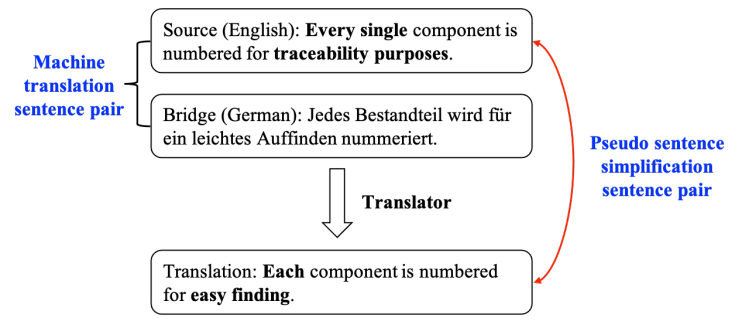

Figure 1: Example of English sentence simplification pair generated by machine translation pair. Large-scale machine translation pairs (e.g. English-German) are chosen as a source base. The sentence of bridge language (e.g. German) is translated into an English sentence. After pairing the results of the source (English) and the translated sentence, we can harvest large-scale pseudo sentence pairs, as the red dashed arrow shows.

SS as a monolingual machine translation task that translating from complex sentences to simplified sentences, whose performance rely heavily on the quality of parallel SS corpus. However, much work (Woodsend and Lapata, 2011; Coster and Kauchak, 2011; Xu et al., 2016; Qiang and Wu, 2021) pointed out that the public English SS benchmark (WikiLarge (Zhang and Lapata, 2017)) which align sentences from English Wikipedia and Simple English Wikipedia are deficient, because they contain a large proportion of inaccurate or inadequate simplifications, which lead to SS methods that generalize poorly. Additionally, parallel SS corpus is difficult to obtain in all languages other than English. Therefore, in the paper, we focus on how to build SS corpora in multiple languages using an unsupervised method.

Some work Kajiwara and Komachi (2018); Martin et al. (2020b) built pseudo parallel corpora by searching the nearest neighbor sentence for each sentence based on embedding model from a largescale text corpus. We can see that the built corpora are more like paraphrase corpora instead of SS corpora. It is because: (1) It only guarantees that the aligned sentences are highly similar, and cannot 
guarantee that the aligned sentences preserve the same meanings; and (2) Each sentence pair does not distinguish between the simple sentence and the complex sentence.

In this paper, we present an unsupervised method to build SS parallel corpora based on a large-scale bilingual translation corpus. Many languages have large-scale training corpora, which lie at the core of the recent success of neural machine translation (NMT) models. If we plan to build a pseudo English SS corpus, the main idea of our method is demonstrated in Figure 1: we use a translator to translate the sentence in a bridge language (e.g., German) into English, and pair them as a pseudo SS sentence pair. The idea is motivated by the following two findings :

(1) NMT models usually tend to generate more high-frequency tokens and less low-frequency tokens (Gu et al., 2020; Jiang et al., 2019). Considering that the higher the word frequency, the more simple the word is, this phenomenon could be beneficial to text simplification (Saggion, 2017; Qiang et al., 2020a).

(2) The difference of text complexity levels exists between the source and target language of translation corpus (Bentz et al., 2016). From the perspective of linguistics, word entropy, morphological complexity, and syntactic complexity vary between languages. A sentence with lower complexity is more likely to be translated into a simpler one.

Each sentence pair in the pseudo SS corpus preserves the same meaning. We measure the difference of sentence complexity between the original sentence and the translated sentence using Flesch reading-ease score (Kincaid et al., 1975), and keep pairs with a higher complexity difference as SS corpus. For each remaining sentence pair, the sentence with a higher score will be treated as the simple sentence and the other sentence as the complex.

The contributions of our paper are as follows:

(1) We propose an unsupervised method to build SS corpora in multiple languages because our method can be used to languages with large-scale NMT resources. Our method can guarantee that the aligned sentences preserve the same meanings and have difference in text complexity levels.

(2) We provide SS corpora in three languages (English, French, and Spanish) to train SS models, alleviating the need for language-specific supervised corpora. We plan on making these resources publicly available after this paper is published ${ }^{1}$.

(3) Experimental results show that SS methods on our English corpus significantly outperform the results on the English SS benchmark (WikiLarge). We adopt pre-trained language modeling BART on our English SS corpus to achieve the state-of-theart in English with 42.69 SARI on ASSET and 41.97 SARI on TURKCORPUS datasets.

\section{Related Work}

\subsection{Supervised Sentence Simplification}

Supervised sentence simplification (SS) methods treat sentence simplification task as monolingual machine translation task that translating from complex sentences to simplified sentences, requiring supervised parallel training corpora of complexsimple aligned sentences (Wubben et al., 2012a; Martin et al., 2020a; Nisioi et al., 2017; Xu et al., 2016; Zhang and Lapata, 2017; Scarton and Specia, 2018; Dong et al., 2019; Qiang et al., 2020b). The above methods have relied on WikiSmall (Zhu et al., 2010) or WikiLarge (Zhang and Lapata, 2017), which aligned sentences from English Wikipedia and Simple English Wikipedia. The two datasets have been criticized (Woodsend and Lapata, 2011; Coster and Kauchak, 2011; Xu et al., 2016; Qiang and $\mathrm{Wu}, 2021)$ because they contain a large proportion of inaccurate simplification (not aligned or only partially aligned) and inadequate simplification (not much simpler than complex sentence). Professional simplifications such as Newsela dataset (Xu et al., 2015) have high-quality sentence pairs. But, it is usually accompanied by restrictive licenses that prevent widespread usage and reproducibility.

Researchers have attempted to design SS methods in other languages such as Spanish (Saggion et al., 2015), Portuguese (Aluísio et al., 2008), Japanese (Goto et al., 2015), French (Gala et al., 2020) and Italian (Brunato et al., 2015). But, these approaches are limited by the availability of parallel SS corpora. In this paper, we propose a general framework that can be used to obtain large-scale SS data for these languages to train neural SS methods.

\subsection{Unsupervised Sentence Simplification}

To overcome the scarcity of parallel SS corpus, unsupervised SS methods without using any parallel corpus have attracted much attention. Existing unsupervised SS methods can be divided into two

\footnotetext{
${ }^{1}$ https://github.com/luxinyu1/Trans-SS
} 
classifications. The first scheme focuses on how to design an unsupervised SS method, and the second scheme concentrates on how to build a parallel SS corpus.

Narayan and Gardent (2015) and Kumar et al. (2020) are the pipeline-based unsupervised framework, where the pipeline of Narayan and Gardent is composed of lexical simplification, sentence splitting, and phrase deletion, the pipeline of Kumar et al. includes deletion, reordering, and lexical simplification. Surya et al. (2019) proposed an unsupervised neural text simplification based on a shared encoder and two decoders, which only learn the neural network parameters from simple sentences set and complex sentences set. In other languages, there are unsupervised statistical machine translations for Japanese (Katsuta and Yamamoto, 2019) and back-translation in Spanish and Italian (Palmero Aprosio et al., 2019). The performance of the above unsupervised SS methods is however often below their supervised counterparts.

Some work (Kajiwara and Komachi, 2018; Martin et al., 2020b) constructed SS corpora by searching the most similar sentences using sentence embedding modeling, and train SS methods using the constructed SS corpora. Kajiwara and Komachi (2018) calculated the similarity between the sentences from English Wikipedia by Word Mover's distance (Kusner et al., 2015). Martin et al. (2020b) adopted multilingual sentence embedding modeling LASER (Artetxe et al., 2018) to calculate the similarity between the sentences from 1 billion sentences from CCNET (Wenzek et al., 2019). Since the aim of the two works is to find the most similar sentences from a large corpus, they cannot guarantee that the aligned sentences preserve the same meanings.

\subsection{Paraphrase Mining}

Some work has focused on generating paraphrase corpus for neural machine translation (NMT) systems using back-translation, where backtranslation (Sennrich et al., 2015) is a technique widely used in NMT to enhance the target monolingual data during the training process. Specifically, the back-translation technique is used by translating the non-English side of bitexts back to English(Wieting et al., 2017) and pairing translations with the references. Two large paraphrase corpora (PARANMT-50M (Wieting and Gimpel, 2017) and PARABANK (Hu et al., 2019)) are built based on this idea, and has been proven to have great potential in different translation-core tasks. Round-trip translation is also used in mining paraphrases (Mallinson et al., 2017) by translating sentences into another language then translating the result back into the original language. Similar to machine translation, back-translation is used to improve the performance of neural SS methods (Katsuta and Yamamoto, 2019; Palmero Aprosio et al., 2019; Qiang and Wu, 2021). Mehta et al. (2020) trained a paraphrasing model by generating a paraphrase corpus using back-translation, which is used to preprocess source sentences of the low-resource language pairs before feeding into the NMT system.

The above work for building a large paraphrase corpus is to serve for NMT and other tasks, which is not fit for SS task. The difference of sentence complexity between the original sentence and the translated sentence for each sentence pair has not been taken into consideration, which is vitally important for SS task. Therefore, we focus on how to build a sentence simplification corpus, instead of a paraphrase corpus.

\section{Method}

In this paper, we present our unsupervised method to build SS corpora in multiple languages, which is motivated by high-frequency tokens generated by NMT modeling and the difference of text complexity levels between bilingual translation corpora. The overall architecture of the proposed method for building the English SS corpus is illustrated in Figure 2. Our method consists of two steps (Paraphrase Generation and Selectors) to build SS corpora, to achieve the following two requirements:

(1) The two sentences of each sentence pair should convey the same meaning. Given highresource parallel machine translation corpus, we can obtain paraphrase corpus by translating the sentences of the bridge language into the target language using a Translator.

(2) The two sentences of each sentence pair should have difference in text complexity levels. After obtaining the paraphrase corpus, we calculate the text complexity of the two sentences using text readability formulas and keep these pairs with a higher complexity difference.

Pseudo SS Generation In contrast to previous work(Wieting and Gimpel, 2017; Hu et al., 2019) mining paraphrases for focusing on lexical and sen- 


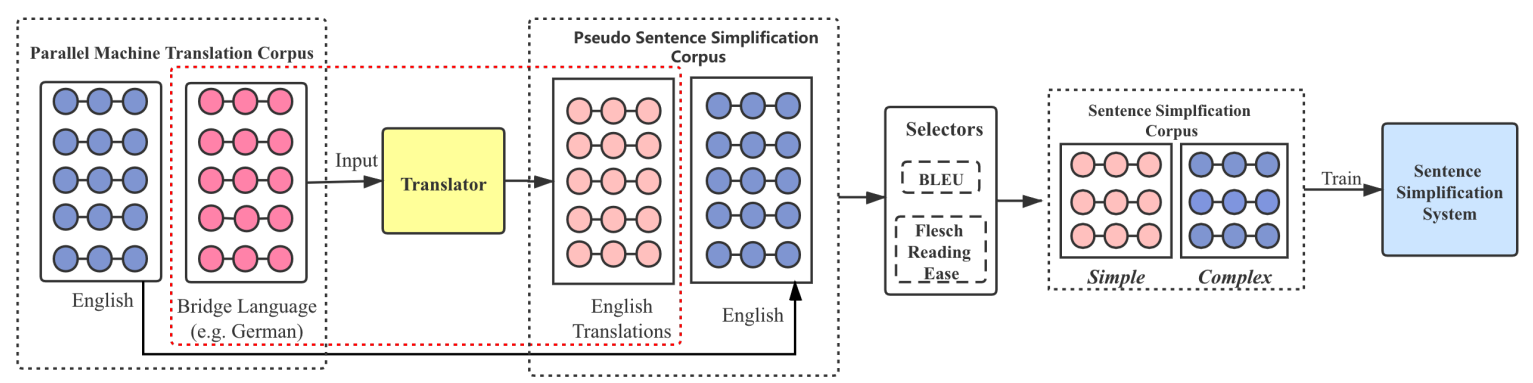

Figure 2: The overview of our approach for Building English SS corpus. Our approach is composed of a highresource bilingual translation corpus and a translator. A pseudo paraphrase corpus is synthesized by pairing the source sentences (English) and the translated sentences of the bridge language. Then, we select these complexsimple sentence pairs with a higher complexity difference, as the sentence simplification corpus.

tence structure diversity, we mine paraphrases as pseudo SS corpus for mainly considering the fluency and syntax correctness.

Specifically, if we plan to construct a SS corpus for a specific language $A$, we need to obtain a highresource bilingual translation corpus of language $A$ and language $B$, and a Translator which can translate the sentences in language $B$ into the language $A$, where $B$ is the bridge language. It should be noted that the chosen bridge language determines the availability of parallel machine translation corpus, and also further determines the performance of the Translator. Therefore, the bridge language $B$ and the specific language $A$ should have a highresource parallel corpus. For example, as shown in Figure 2, we choose German as a bridge language for building the English SS corpus.

Selectors In this step, we design a simple pipeline consisting of only two selectors (BLEU and Flesch Reading Ease) for selecting some sentence pairs with a higher complexity difference.

Firstly, we select these pairs with the BLEU scores $^{2}$ above a threshold $h_{B L E U}$, for ensuring the quality of paraphrases. It is worthy to note that, this selector is mainly used to filter the unaligned sentence pairs in paraphrase corpus because translation mistakes are relatively rare owing to recent improvements on NMT models. Meanwhile, we filter out the translations which are the same as references.

Secondly, we measure the difference of text complexity using Flesch reading ease score (FRES) (Kincaid et al., 1975), which is designed to indicate how difficult a sentence is to understand, and

\footnotetext{
${ }^{2}$ The BLEU scores in this work are calculated by SACREBLEU (Post, 2018)
}

is widely used to evaluate the performance of SS. FRES grades the text from 0 to 100 . The higher scores indicate the sentences are easier to read. As usual, the difference of one school grade level in FRES is 10, e.g., 5th grade (100.00-90.00) and 6th grade (90.0-80.0). The formula of FRES is,

$$
k_{1}-k_{2}\left(\frac{\# \text { words }}{\# \text { sentences }}\right)-k_{3}\left(\frac{\# \text { syllables }}{\# \text { words }}\right)
$$

Here $k_{1}, k_{2}, k_{3}$ are coefficients that vary in different languages, which are set by linguists. The parameters of FRES in English are set to $k_{1}=206.835, k_{2}=1.015, k_{3}=84.6$.

To ensure simplicity, we only keep the sentence pairs with a FRES difference higher than a threshold $h_{F R E S}$. In our experiments, we set $h_{B L E U}=$ 15.0 and $h_{F R E S}=10.0$, where $h_{F K E}=10.0$ means that for each sentence pair, the simplified version should be at least one school level simpler than its its unsimplified counterpart.

\section{Sentence Simplification Corpora}

Our unsupervised method can be used to languages with large-scale bilingual translation corpora. According to this principle, we choose the three languages (English, French, and Spanish) to build SS corpora, to train SS systems.

English In the step of paraphrase generation, we choose German as bridge language and obtains the bilingual translation corpus provided by huggingface ${ }^{3}$ with $4,000,000$ sentence pairs as De-En. We use Facebook FAIR's state-of-the-art $D e \rightarrow E n$

\footnotetext{
de.tgz

${ }^{3} \mathrm{https} / / / \mathrm{cdn}$-datasets.huggingface.co/translation/wmt_en_
} 


\begin{tabular}{l|l}
\hline $\begin{array}{l}\text { Bridge Language } \\
\text { Complex } \\
\text { Simple }\end{array}$ & $\begin{array}{l}\text { Er sagt er bekomme Platzangst und fuehle sich, als ob er in einem Sarg begraben werde. } \\
\text { He says he gets claustrophobic, that he feels trapped as if he was buried in a coffin. } \\
\text { He says he gets scared and feels like he's being buried in a coffin. }\end{array}$ \\
\hline $\begin{array}{l}\text { Bridge Language } \\
\text { Complex } \\
\text { Simple }\end{array}$ & $\begin{array}{l}\text { Das Hotel Gates am Kudamm, mit seiner einmaligen Gastfreundschaft, müssen Sie unbedingt einmal selbst erleben. } \\
\text { You simply must experience the Hotel Gates Am Kudamm with its unique concept of hospitality. } \\
\text { The Hotel Gates Am Kudamm, with its unique hospitality, is a must-see. }\end{array}$ \\
$\begin{array}{ll}\text { Bridge Language } \\
\text { Complex }\end{array}$ & $\begin{array}{l}\text { Das Geld muss in Unternehmen investiert werden, die garantieren, dass Hochschulabgänger einen Arbeitsplatz finden. } \\
\text { Simple money must be invested in enterprises which guarantee that graduates will find employment. } \\
\text { The money must be invested in companies that guarantee that graduates will find a job. }\end{array}$ \\
\hline
\end{tabular}

Table 1: Examples of English SS corpus generated by our method. The differences between the complex sentence and the simple sentence are emphasized in bold .

\begin{tabular}{l|c|ccc}
\hline & WikiLarge & English & French & Spanish \\
\hline Vocab(complex) & 169,349 & 196,301 & 112,335 & 119,876 \\
Vocab(simple) & 135,607 & 165,130 & 102,672 & 104,361 \\
\hline Avg(complex) & 21.93 & 18.95 & 26.17 & 28.00 \\
Avg(simple) & 16.14 & 19.36 & 27.74 & 25.79 \\
\hline Total pairs & 296,402 & 816,058 & 621,937 & 487,862 \\
\hline
\end{tabular}

621,937 , and 48,7862, respectively. Because the SS task is a paraphrase generation task using easier words, the length of the complex sentence and the simple sentence are roughly the same, and the size of the vocabulary in the simple sentence set should be smaller than the complex sentence set. From

Table 2: Statistics of our building corpora in English, French, and Spanish compared with Wikilarge. Avg(complex) and Avg(simple) are the average numbers of words in the complex sentences and the simpler sentences, respectively.

model $^{4}(\mathrm{Ng}$ et al., 2019) as the translator, which is based on big Transformer(Vaswani et al., 2017) architecture training on WMT19 dataset.

French and Spanish For both French and Spanish, English is chosen as the bridge language. The bilingual translation corpora for the two languages are from the full Europarl-v7 dataset ${ }^{5}$, where the sentence pairs of English-French and EnglishSpanish are 1,965,734 and 2,007,723, respectively.

For French, the translator from English to French is also a Transformer-based model ${ }^{6}(\mathrm{Ott}$ et al., 2018). For Spanish, the translator from English to Spanish is fine-tuned by pre-trained language model mBART(Liu et al., 2020) ${ }^{7}$.

The parameters of FRES in French is set to $k_{1}=$ $207, k_{2}=1.015, k_{3}=73.6$, and the parameters in German are $k_{1}=180, k_{2}=58.5, k_{3}=1.0$.

Statistics and Examples We show some examples of the sentence pairs generated by our method in Table 1. We report the statistics of our building corpora in Table 2. The numbers of sentence pairs in English, French, and Spanish are 816,058,

\footnotetext{
${ }^{4}$ https://dl.fbaipublicfiles.com/fairseq/models/wmt19.ende.joined-dict.ensemble.tar.gz

${ }^{5}$ https://www.statmt.org/europarl/

${ }^{6}$ https://dl.fbaipublicfiles.com/fairseq/models/wmt14.enfr.joined-dict.transformer.tar.bz2

${ }^{7}$ https://dl.fbaipublicfiles.com/fairseq/models/mbart/mbart .cc25.v2.tar.gz
}

Table 2, we can see that our three corpora satisfy the expectations of the SS task. In contrast to our corpora, the length of the complex sentence in WikiLarge is longer than the simple sentence, because it focuses on the deletion of content.

\section{Experiments}

We design experiments to answer the following two questions:

Q1. Effectiveness: Is the English SS corpus built by our method a better dataset compared with the benchmark WikiLarge and the dataset built by (Martin et al., 2020b)?

Q2. Universality: Can our unsupervised method be used to build SS corpora for other languages with large-scale bilingual translation corpora?

\subsection{Evaluation Datasets}

We choose four datasets to evaluate the performance of SS modelings on our corpora: two datasets for English (TURKCORPUS and ASSET), ALECTOR for French, and SIMPLEXT for Spanish. The statistics are reported in Table 4.

For evaluating English simplification task, we use two widely used evaluation benchmarks TURKCORPus (Xu et al., 2016) and ASSET (AlvaManchego et al., 2020) of WikiLarge dataset. Both TURKCORPUS and its improved version ASSET consist of 2,000 valid sentences and 359 test sentences. Each original sentence in TURKCORPUS has 8 simplification references collected through Amazon Mechanical Turk. ASSET with 10 simplification references per original sentence focuses on multiple simplification operations including lexical 


\begin{tabular}{|c|c|c|c|c|c|c|c|}
\hline & \multirow[t]{2}{*}{ Data } & \multicolumn{3}{|c|}{ TURKCORPUS } & \multicolumn{3}{|c|}{ ASSET } \\
\hline & & SARI $\uparrow$ & FKGL $\downarrow$ & BLEU $\uparrow$ & $\mathrm{SARI} \uparrow$ & FKGL $\downarrow$ & BLEU $\uparrow$ \\
\hline Source & - & 26.29 & 10.02 & 99.36 & 20.73 & 10.02 & 92.81 \\
\hline Reference & - & 40.21 & 8.73 & 73.00 & 45.14 & 6.48 & 70.12 \\
\hline PBMT-R(Wubben et al., 2012b) & WikiSmall & 38.04 & 8.85 & 82.49 & 34.63 & 8.85 & 79.39 \\
\hline Dress-LS(Zhang and Lapata, 2017) & WikiLarge & 36.97 & 7.66 & 81.08 & 36.59 & 7.66 & 86.39 \\
\hline DMASS-DCSS(Zhao et al., 2018) & WikiLarge & 39.92 & 7.73 & 73.29 & 38.67 & 7.73 & 71.44 \\
\hline ACCESS(Martin et al., 2020a) & WikiLarge & 41.38 & 7.29 & 76.36 & 40.13 & 7.29 & 75.99 \\
\hline UNTS(Surya et al., 2019) & Unsupervised & 36.29 & 7.60 & 76.44 & 35.19 & 7.60 & 76.14 \\
\hline BTTS10(Kumar et al., 2020) & Unsupervised & 36.91 & 7.83 & 82.00 & 35.72 & 7.83 & 83.01 \\
\hline \multirow[t]{2}{*}{ LSTM } & WikiLarge & 35.69 & 7.7 & 79.45 & 35.81 & 6.06 & 72.3 \\
\hline & Ours & 38.21 & 8.41 & 76.85 & 37.65 & 7.97 & 71.71 \\
\hline \multirow[t]{2}{*}{ ConvS2S } & WikiLarge & 36.83 & 7.58 & 80.40 & 36.48 & 7.18 & 82.29 \\
\hline & Ours & 38.98 & 8.66 & 73.79 & 37.92 & 7.89 & 69.67 \\
\hline \multirow[t]{3}{*}{ Transformer } & Wikilarge & 37.05 & 8.42 & 86.71 & 34.16 & 8.42 & 84.47 \\
\hline & MUSS(Martin et al., 2020b) & 38.06 & 9.43 & 63.70 & 38.03 & 9.41 & 61.76 \\
\hline & Ours & 39.99 & 7.97 & 72.75 & 39.58 & 7.83 & 70.81 \\
\hline \multirow[t]{3}{*}{ BART } & WikiLarge & 38.96 & 8.15 & 84.58 & 36.81 & 8.15 & 85.66 \\
\hline & MUSS(Martin et al., 2020b) & - & - & - & 39.73 & 9.26 & 65.00 \\
\hline & Ours & 41.97 & 8.21 & 73.72 & 42.69 & 7.94 & 71.83 \\
\hline
\end{tabular}

Table 3: Results of English sentence simplification. $\uparrow$ The higher, the better. $\downarrow$ The lower, the better. - indicates the results that are not found in the original paper.

\begin{tabular}{lcccc}
\hline & Lang. & \#Valid & \#Test & C.R. \\
\hline TURKCORPUS & English & 2000 & 359 & 0.95 \\
ASSET & English & 2000 & 359 & 0.83 \\
ALECTOR & French & 800 & 801 & 0.97 \\
SIMPLEXT & Spanish & 708 & 708 & 0.48 \\
\hline
\end{tabular}

Table 4: The statistics of SS evaluation datasets. C.R.(Compression Ratio) is the amount of compression of the complex sentence relative to the simple sentence.

paraphrasing, compression, and sentence splitting.

For French, we use ALECTOR (Gala et al., 2020) for evaluation, which contains 1601 sentence pairs. It contains 79 original literary and scientific texts along with their simplified equivalents, which are chosen from materials for French Primary school students. We split it into a valid set (first 800 pairs) and a test set (next 801 pairs).

For Spanish, we use SIMPLEXT (Saggion et al., 2015; Saggion, 2017) for evaluation, which contains 1416 sentence pairs. It is from 200 news articles that were manually simplified by experienced experts for people with learning disabilities. We split it into a valid set (708 pairs) and a test set (708 pairs).

\subsection{Training Details}

To compare the quality of our building corpora with other training datasets, we test the following four models: LSTM-based, ConvS2S-based,
Transformer-based, and BART-based models. We implement the four models via fairseq(Ott et al., 2019). The parameters of all these models are tuned with SARI on validation sets. The parameters of the four models are shown below.

We adopt the Adam optimizer with $\beta_{1}=$ $0.9, \beta_{2}=0.999, \epsilon=10^{-8}$ for LSTMbased, Transformer-based and BART-based models, the NAG optimizer for ConvS2S-based model. Dropout is set 0.1 for LSTM-based, ConvS2Sbased and BART-based models and 0.2 for Transformer-based model. The initial learning rate are set to $5 \times 10^{-4}, 3 \times 10^{-4}, l r=3 \times 10^{-5}$ for LSTM-based, Transformer-based and BART(large )-based models, respectively. We use a fixed learning rate of $l r=0.5$ for ConvS2S-based model. Byte Pair Encoding(BPE) is used in all the models for word segmentation.

For BART-based model used for English SS, we initialize the model with the pretrained weights ${ }^{8}$. For BART-based model used for French and Spanish languages, we adopt a multilingual pretrained BART (mBART) with the weights ${ }^{7}$ pretrained on 25 languages.

\subsection{Evaluation Metrics}

SARI(Xu et al., 2016) is the main metric to evaluate text simplification models, which calculates the

\footnotetext{
${ }^{8}$ https://dl.fbaipublicfiles.com/fairseq/models/bart.large.ta r.gz
} 
arithmetic mean of the $n$-gram F1 scores of three operations (keeping, adding, and deleting) through comparing the generated sentences to multiple simplification references and the original sentences.

Flesch-Kincaid Grade Level (FKGL)(Kincaid et al., 1975) based on FRES (Formula 1) is widely used to evaluate the SS task, which measures the readability of the system output.

Earlier work also used BLEU (Papineni et al., 2002) as a metric, but recent work has found that it does not correlate with simplicity (Sulem et al., 2018). Systems with high BLEU scores are thus biased towards copying the original sentences as a whole (e.g., 99.36 on TURKCORPUS or 92.81 on ASSET). For completeness, we also report BLEU scores. For the above metrics, we use standard simplification evaluation tool $\mathrm{EASSE}^{9}$ to compute their scores.

\subsection{English Simplification}

We choose four supervised SS methods (PBMTR(Wubben et al., 2012b), Dress-LS(Zhang and Lapata, 2017), DMASS-DCSS(Zhao et al., 2018), and ACCESS(Martin et al., 2020a) ), two unsupervised SS methods (UNTS(Surya et al., 2019) and BTTS10(Kumar et al., 2020)) to compare. We also choose the SS corpus built by (Martin et al., 2020b) as a comparison.

Table 3 summarizes the evaluation results of SS methods on our building English corpus. We first compare the results between our building English SS corpus and English SS corpus WikiLarge. In terms of SARI metric, we can see that the four models (LSTM-based, ConvS2S, Transformer-based, and BART-based) on our data significantly outperform the results on WikiLarge dataset, demonstrating the promise of building SS corpora using our method. BART-based method achieves the best results compared with the other three models (LSTMbased, ConvS2S, and Transformer-based). On TURKCORPUS and ASSET, BART-based method on our data significantly outperforms the results on WikiLarge by a large margin $(+3.01,+5.88$ SARI $)$. In terms of readability, BART on our data obtains lower (=better) FKGL compared to the results on WikiLarge. We believe this improvement shows that our method for building English SS corpus is a good choice for SS task.

We then compare the results between our building corpus and MUSS build by (Martin et al.,

\footnotetext{
${ }^{9}$ https://github.com/feralvam/easse
}

2020b). Compare with MUSS, SS methods on our dataset outperform the results on MUSS in terms of all the measurements. Transformer-based method on our dataset achieves a large improvement of $(+1.93,+1.55$ SARI $)$ on TURKCORPUS and AS SET, and BART-based method achieves a large margin of +2.96 SARI on ASSET. In terms of readability, Transformer-based and BART-based methods obtain lower FKGL compared with MUSS, which indicated the output of the SS methods is easier to understand. These indicate that the effectiveness of our method on building SS English corpus.

\subsection{French and Spanish Simplification}

\begin{tabular}{llcccc}
\hline & \multirow{2}{*}{ Data } & \multicolumn{2}{c}{ ALECTOR } & \multicolumn{2}{c}{ SIMPLEXT } \\
& & SARI $\uparrow$ & FRES $\uparrow$ & SARI $\uparrow$ & FRES $\uparrow$ \\
\hline Source & - & 26.36 & 66.57 & 5.65 & 49.40 \\
Pivot & - & 38.52 & $\mathbf{6 5 . 5 5}$ & 25.98 & 56.30 \\
\hline mBART+ & MUSS & 38.35 & 68.36 & 19.81 & 55.07 \\
\hline Transformer & Ours & 36.93 & 75.56 & $\mathbf{3 0 . 3 7}$ & 44.51 \\
mBART & Ours & $\mathbf{3 9 . 0 0}$ & 73.15 & 27.83 & $\mathbf{4 7 . 3 0}$ \\
\hline
\end{tabular}

Table 5: Results of unsupervised sentence simplification in French and Spanish. We choose FRES metric instead of its revision FKGL in French and Spanish because the coefficients of FKGL in these two languages are not available.

Our approach can be applied to any language owing to large-scale translation corpora. Different from English SS task, large-scale SS training corpus in other languages is hard to obtain. For a better comparison, we add one new baseline (Pivot) via machine translation. Specifically, for Pivot, give one non-English sentence, we translate the sentence to English and translate the translated sentence back into the source language. Here, we use Google Translator ${ }^{10}$ for French and Spanish translation. We also choose the best results of mBART+ (mBART+ACCESS) on MUSS dataset (Martin et al., 2020b), where ACCESS (Martin et al., 2020a) is a control mechanism to the parameters of SS model by controlling attributes such as length, lexical complexity, and syntactic complexity.

The results are shown in Table 5. We can see that the same SS methods on our dataset outperform the results on MUSS, which verifies that our method is more fit for SS task. Compared with the results of Pivot, SS methods on our building SS corpora can

\footnotetext{
${ }^{10} \mathrm{https}: / /$ translate.google.com/
} 
generate more simplified sentences. We conclude that our method for building SS corpora can be used to languages with large-scale bilingual translation corpora.

\subsection{Ablation Study of our method}

To further analyze the factors affecting our unsupervised method for building SS corpus, we do more experiments in this subsection.

\begin{tabular}{llcccc}
\hline & \multirow{2}{*}{ Method } & \multicolumn{2}{c}{ TURKCORPUS } & \multicolumn{2}{c}{ ASSET } \\
& & SARI $\uparrow$ & FKGL $\downarrow$ & SARI $\uparrow$ & FKGL $\downarrow$ \\
\hline \multirow{2}{*}{ Pseduo SS } & Transformer & 34.18 & 9.49 & 29.46 & 9.49 \\
& BART & 33.94 & 9.72 & 29.92 & 9.72 \\
\hline \multirow{2}{*}{ w/o BLEU } & Transformer & 38.53 & 6.37 & 39.05 & 6.08 \\
& BART & 38.61 & 7.01 & 40.62 & 6.54 \\
\multirow{2}{*}{ wo FRES } & Transformer & 34.86 & 9.88 & 30.49 & 9.63 \\
& BART & 35.97 & 9.69 & 31.54 & 9.69 \\
\hline \multirow{2}{*}{ full } & Transformer & 39.99 & 7.97 & 39.58 & 7.83 \\
& BART & 41.97 & 8.21 & 42.69 & 7.64 \\
\hline
\end{tabular}

Table 6: Ablation study results of SS methods on our English corpus without BLEU selector and FRES selector. "w/o" denotes "without".

(1) Influence of each selector in our method. To evaluate the effect of each selector in our methods, we build four different SS corpus: pseudo SS corpus, the corpus building by our method without BLEU selector, the corpus building by our method without FRES selector, and the corpus building by our full method. We choose two SS methods (Transformer-based and BART-based) to do the experiments, and the results show in Table 6. It is very obvious that the results on pseudo SS corpus are the worst and our method combing two selectors achieves the best results. FRES selector in our method is more important than BLEU selector, because FRES selector is used to select the sentence pairs with a higher complexity difference and BLEU selector is only used to filter the unaligned sentence pairs.

(2) Influence of the size of the corpus built by our method. Because the size of our SS corpus is 816,058 and the size of WikiLarge is 296,402 , we vary the size from $10 \mathrm{~K}$ to $800 \mathrm{~K}$ to analyze the results of Transformer-based on the two corpora. Due to the size of WikiLarge, we only show the results of WikiLarge in the first 30K samples. We can see that the SARI values increase at first and keep stable finally when increasing the size of training samples. We see that the size of the SS corpus is of vital importance for SS methods. In the paper, we only choose a bilingual translation corpus of size
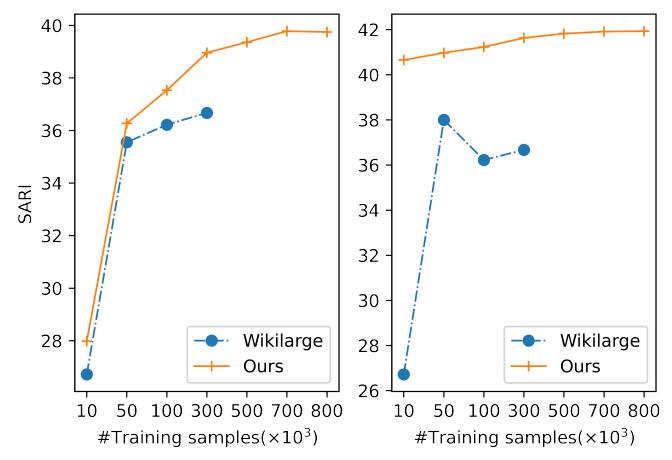

Figure 3: The performance of Transformer-based and BART-based method on TURKCORPUS when varying the size of the corpus.

$4,000,000$. In the future, we will try to build SS corpora using a more large-scale bilingual translation corpus.

\subsection{Qualitative Study}

Table 7 shows some simplified sentences from the test set of TURKCORPUS by our method BARTbased method trained with our building English corpus. Our model reduces more linguistic complexity of the source sentence, while still retaining its original information and meaning. We can found that our method more focuses on lexical simplification, e.g., "stomach" as a simpler for "inoperable abdominal", "very" as a simpler for "extremely", etc. We draw the same conclusions from these examples that our building method can be used to train SS methods.

\section{Conclusions}

We propose an unsupervised method to build large parallel corpora for training sentence simplification (SS) models. Our method consists of a highresource bilingual translation corpus and a translator. Unsupervised SS models can be trained by pairing the source sentences in the bilingual translation corpus and the translated sentences of the bridge language generated by the translator. We conduct experiments and show that SS models trained on synthetic data generated by our approach significantly outperform the results on English benchmark WikiLarge. In the future, we plan to investigate the influence of different text readability methods.

\section{Acknowledgements}

This research is partially supported by the National Natural Science Foundation of China under grants 


\begin{tabular}{ll}
\hline $\begin{array}{l}\text { Source } \\
\text { Reference } \\
\text { Ours }\end{array}$ & $\begin{array}{l}\text { He was diagnosed with inoperable abdominal cancer in April } 1999 . \\
\text { He was diagnosed with abdominal cancer in April 1999. } \\
\text { He was diagnosed with stomach cancer in April 1999. }\end{array}$ \\
\hline $\begin{array}{l}\text { Source } \\
\text { Reference }\end{array}$ & $\begin{array}{l}\text { Heavy rain fell across portions of Britain on October 5, causing localized accumulation of flood waters. } \\
\text { Heavy rain fell across Britain on October 5, causing accumulation of flood waters. }\end{array}$ \\
Ours & Heavy rain fell on parts of the UK on October 5, causing localized flooding. \\
\hline Source & Admission to Tsinghua is extremely competitive. \\
Reference & Admission to Tisinghua is competitive. \\
Ours & Admission to Tsinghua is very competitive. \\
\hline Source & They are culturally akin to the coastal peoples of Papua New Guinea. \\
Reference & They are similar to the coastal peoples of Papua New Guinea. \\
Ours & They are similar in culture to the coastal peoples of Papua New Guinea. \\
\hline
\end{tabular}

Table 7: Examples of simplifications generated by BART-based method on our building English corpus. The bold words highlight the differences.

\section{7 and 61906060.}

\section{References}

Sandra M Aluísio, Lucia Specia, Thiago AS Pardo, Erick G Maziero, and Renata PM Fortes. 2008. Towards brazilian portuguese automatic text simplification systems. In Proceedings of the eighth ACM symposium on Document engineering, pages 240-248.

Fernando Alva-Manchego, Louis Martin, Antoine Bordes, Carolina Scarton, Benoît Sagot, and Lucia Specia. 2020. ASSET: A dataset for tuning and evaluation of sentence simplification models with multiple rewriting transformations. In Proceedings of the 58th Annual Meeting of the Association for Computational Linguistics, pages 4668-4679, Online. Association for Computational Linguistics.

Mikel Artetxe, Gorka Labaka, and Eneko Agirre. 2018. Unsupervised statistical machine translation. In Proceedings of the 2018 Conference on Empirical Methods in Natural Language Processing, pages 3632 3642, Brussels, Belgium. Association for Computational Linguistics.

Christian Bentz, Tatjana Soldatova, Alexander Koplenig, and Tanja Samardžić. 2016. A comparison between morphological complexity measures: typological data vs. language corpora.

Dominique Brunato, Felice Dell'Orletta, Giulia Venturi, and Simonetta Montemagni. 2015. Design and annotation of the first italian corpus for text simplification. In Proceedings of The 9th Linguistic Annotation Workshop, pages 31-41.

John Carroll, Guido Minnen, Yvonne Canning, Siobhan Devlin, and John Tait. 1998. Practical simplification of english newspaper text to assist aphasic readers. In Proceedings of the AAAI-98 Workshop on Integrating Artificial Intelligence and Assistive Technology, pages 7-10.
Raman Chandrasekar, Christine Doran, and Srinivas Bangalore. 1996. Motivations and methods for text simplification. In COLING 1996 Volume 2: The 16th International Conference on Computational Linguistics, pages 1041-1044.

William Coster and David Kauchak. 2011. Learning to simplify sentences using wikipedia. In Proceedings of the workshop on monolingual text-to-text generation, pages 1-9.

Yue Dong, Zichao Li, Mehdi Rezagholizadeh, and Jackie Chi Kit Cheung. 2019. EditNTS: An neural programmer-interpreter model for sentence simplification through explicit editing. In Proceedings of the 57th Annual Meeting of the Association for Computational Linguistics, pages 3393-3402, Florence, Italy. Association for Computational Linguistics.

Richard Evans, Constantin Orasan, and Iustin Dornescu. 2014. An evaluation of syntactic simplification rules for people with autism. Association for Computational Linguistics.

Núria Gala, Anaïs Tack, Ludivine Javourey-Drevet, Thomas François, and Johannes C Ziegler. 2020. Alector: A parallel corpus of simplified french texts with alignments of misreadings by poor and dyslexic readers. In Language Resources and Evaluation for Language Technologies (LREC).

Isao Goto, Hideki Tanaka, and Tadashi Kumano. 2015. Japanese news simplification: Task design, data set construction, and analysis of simplified text. Proceedings of MT Summit XV, 1:17-31.

Shuhao Gu, Jinchao Zhang, Fandong Meng, Yang Feng, Wanying Xie, Jie Zhou, and Dong Yu. 2020. Token-level adaptive training for neural machine translation. In Proceedings of the 2020 Conference on Empirical Methods in Natural Language Processing (EMNLP), pages 1035-1046, Online. Association for Computational Linguistics.

J Edward Hu, Rachel Rudinger, Matt Post, and Benjamin Van Durme. 2019. Parabank: Monolingual 
bitext generation and sentential paraphrasing via lexically-constrained neural machine translation. In Proceedings of the AAAI Conference on Artificial Intelligence, volume 33, pages 6521-6528.

Shaojie Jiang, Pengjie Ren, Christof Monz, and Maarten de Rijke. 2019. Improving neural response diversity with frequency-aware cross-entropy loss. In The World Wide Web Conference, pages 28792885.

Tomoyuki Kajiwara and M Komachi. 2018. Text simplification without simplified corpora. The Journal of Natural Language Processing, 25:223-249.

Akihiro Katsuta and Kazuhide Yamamoto. 2019. Improving text simplification by corpus expansion with unsupervised learning. In 2019 International Conference on Asian Language Processing (IALP), pages 216-221. IEEE.

J Peter Kincaid, Robert P Fishburne Jr, Richard L Rogers, and Brad S Chissom. 1975. Derivation of new readability formulas (automated readability index, fog count and flesch reading ease formula) for navy enlisted personnel. Technical report, Naval Technical Training Command Millington TN Research Branch.

Dhruv Kumar, Lili Mou, Lukasz Golab, and Olga Vechtomova. 2020. Iterative edit-based unsupervised sentence simplification. In Proceedings of the 58th Annual Meeting of the Association for Computational Linguistics, pages 7918-7928, Online. Association for Computational Linguistics.

Matt Kusner, Yu Sun, Nicholas Kolkin, and Kilian Weinberger. 2015. From word embeddings to document distances. In International conference on machine learning, pages 957-966. PMLR.

Yinhan Liu, Jiatao Gu, Naman Goyal, Xian Li, Sergey Edunov, Marjan Ghazvininejad, Mike Lewis, and Luke Zettlemoyer. 2020. Multilingual denoising pre-training for neural machine translation. Transactions of the Association for Computational Linguistics, 8:726-742.

Jonathan Mallinson, Rico Sennrich, and Mirella Lapata. 2017. Paraphrasing revisited with neural machine translation. In Proceedings of the 15th Conference of the European Chapter of the Association for Computational Linguistics: Volume 1, Long Papers, pages $881-893$.

Louis Martin, Éric de la Clergerie, Benoît Sagot, and Antoine Bordes. 2020a. Controllable sentence simplification. In Proceedings of the 12th Language Resources and Evaluation Conference, pages 46894698, Marseille, France. European Language Resources Association.

Louis Martin, Angela Fan, Éric de la Clergerie, Antoine Bordes, and Benoît Sagot. 2020b. Multilingual unsupervised sentence simplification. arXiv preprint arXiv:2005.00352.
Sneha Mehta, Bahareh Azarnoush, Boris Chen, Avneesh Saluja, Vinith Misra, Ballav Bihani, and Ritwik Kumar. 2020. Simplify-then-translate: Automatic preprocessing for black-box machine translation.

S. Narayan and C. Gardent. 2015. Unsupervised sentence simplification using deep semantics. pages 111-120.

Nathan Ng, Kyra Yee, Alexei Baevski, Myle Ott, Michael Auli, and Sergey Edunov. 2019. Facebook fair's wmt19 news translation task submission. arXiv preprint arXiv: 1907.06616.

Sergiu Nisioi, Sanja Štajner, Simone Paolo Ponzetto, and Liviu P Dinu. 2017. Exploring neural text simplification models. In Proceedings of the 55th annual meeting of the association for computational linguistics (volume 2: Short papers), pages 85-91.

Myle Ott, Sergey Edunov, Alexei Baevski, Angela Fan, Sam Gross, Nathan Ng, David Grangier, and Michael Auli. 2019. fairseq: A fast, extensible toolkit for sequence modeling. In Proceedings of the 2019 Conference of the North American Chapter of the Association for Computational Linguistics (Demonstrations), pages 48-53, Minneapolis, Minnesota. Association for Computational Linguistics.

Myle Ott, Sergey Edunov, David Grangier, and Michael Auli. 2018. Scaling neural machine translation. In Proceedings of the Third Conference on Machine Translation: Research Papers, pages 1-9, Brussels, Belgium. Association for Computational Linguistics.

Alessio Palmero Aprosio, Sara Tonelli, Marco Turchi, Matteo Negri, and A Di Gangi Mattia. 2019. Neural text simplification in low-resource conditions using weak supervision. In Workshop on Methods for Optimizing and Evaluating Neural Language Generation (NeuralGen), pages 37-44. Association for Computational Linguistics (ACL).

Kishore Papineni, Salim Roukos, Todd Ward, and WeiJing Zhu. 2002. Bleu: a method for automatic evaluation of machine translation. In Proceedings of the 40th annual meeting of the Association for Computational Linguistics, pages 311-318.

Matt Post. 2018. A call for clarity in reporting bleu scores. In Proceedings of the Third Conference on Machine Translation: Research Papers, pages 186191.

Jipeng Qiang, Yun Li, Yi Zhu, Yunhao Yuan, and Xindong $\mathrm{Wu}$. 2020a. Lexical simplification with pretrained encoders. Thirty-Fourth AAAI Conference on Artificial Intelligence, page 8649-8656.

Jipeng Qiang and Xindong Wu. 2021. Unsupervised statistical text simplification. IEEE Transactions on Knowledge and Data Engineering, 33(4):18021806. 
Jipeng Qiang, Qian Zhenyu, Yun Li, Yunhao Yuan, and Xindong Wu. 2020b. Short text topic modeling techniques, applications, and performance: A survey. IEEE Transactions on Knowledge and Data Engineering, page DOI:10.1109/TKDE.2020.2992485.

Luz Rello, Ricardo Baeza-Yates, Stefan Bott, and Horacio Saggion. 2013. Simplify or help? text simplification strategies for people with dyslexia. In Proceedings of the 10th International Cross-Disciplinary Conference on Web Accessibility, pages 1-10.

Horacio Saggion. 2017. Automatic text simplification. Synthesis Lectures on Human Language Technologies, 10(1):1-137.

Horacio Saggion, Sanja Štajner, Stefan Bott, Simon Mille, Luz Rello, and Biljana Drndarevic. 2015. Making it simplext: Implementation and evaluation of a text simplification system for spanish. ACM Transactions on Accessible Computing (TACCESS), $6(4): 1-36$.

Carolina Scarton and Lucia Specia. 2018. Learning simplifications for specific target audiences. In Proceedings of the 56th Annual Meeting of the Association for Computational Linguistics (Volume 2: Short Papers), pages 712-718.

Rico Sennrich, Barry Haddow, and Alexandra Birch 2015. Improving neural machine translation models with monolingual data. arXiv preprint arXiv:1511.06709.

Elior Sulem, Omri Abend, and Ari Rappoport. 2018. BLEU is not suitable for the evaluation of text simplification. In Proceedings of the 2018 Conference on Empirical Methods in Natural Language Processing, pages 738-744, Brussels, Belgium. Association for Computational Linguistics.

Sai Surya, Abhijit Mishra, Anirban Laha, Parag Jain, and Karthik Sankaranarayanan. 2019. Unsupervised neural text simplification. In Proceedings of the 57th Annual Meeting of the Association for Computational Linguistics, pages 2058-2068, Florence, Italy. Association for Computational Linguistics.

Ashish Vaswani, Noam Shazeer, Niki Parmar, Jakob Uszkoreit, Llion Jones, Aidan N Gomez, Lukasz Kaiser, and Illia Polosukhin. 2017. Attention is all you need. arXiv preprint arXiv:1706.03762.

Guillaume Wenzek, Marie-Anne Lachaux, Alexis Conneau, Vishrav Chaudhary, Francisco Guzmán, Armand Joulin, and Edouard Grave. 2019. Ccnet: Extracting high quality monolingual datasets from web crawl data. CoRR, abs/1911.00359.

John Wieting and Kevin Gimpel. 2017. Paranmt-50m: Pushing the limits of paraphrastic sentence embeddings with millions of machine translations. arXiv preprint arXiv:1711.05732.
John Wieting, Jonathan Mallinson, and Kevin Gimpel. 2017. Learning paraphrastic sentence embeddings from back-translated bitext. arXiv preprint arXiv:1706.01847.

Kristian Woodsend and Mirella Lapata. 2011. Learning to simplify sentences with quasi-synchronous grammar and integer programming. In Proceedings of the 2011 Conference on Empirical Methods in Natural Language Processing, pages 409-420.

Sander Wubben, EJ Krahmer, and APJ van den Bosch. 2012a. Sentence simplification by monolingual machine translation.

Sander Wubben, Antal van den Bosch, and Emiel Krahmer. 2012b. Sentence simplification by monolingual machine translation. In Proceedings of the 50th Annual Meeting of the Association for Computational Linguistics (Volume 1: Long Papers), pages 10151024, Jeju Island, Korea. Association for Computational Linguistics.

Wei Xu, Chris Callison-Burch, and Courtney Napoles. 2015. Problems in current text simplification research: New data can help. Transactions of the Association for Computational Linguistics, 3:283-297.

Wei Xu, Courtney Napoles, Ellie Pavlick, Quanze Chen, and Chris Callison-Burch. 2016. Optimizing statistical machine translation for text simplification. Transactions of the Association for Computational Linguistics, 4:401-415.

Xingxing Zhang and Mirella Lapata. 2017. Sentence simplification with deep reinforcement learning. In Proceedings of the 2017 Conference on Empirical Methods in Natural Language Processing, pages 584-594, Copenhagen, Denmark. Association for Computational Linguistics.

Sanqiang Zhao, Rui Meng, Daqing He, Andi Saptono, and Bambang Parmanto. 2018. Integrating transformer and paraphrase rules for sentence simplification. In Proceedings of the 2018 Conference on Empirical Methods in Natural Language Processing, pages 3164-3173, Brussels, Belgium. Association for Computational Linguistics.

Zhemin Zhu, Delphine Bernhard, and Iryna Gurevych. 2010. A monolingual tree-based translation model for sentence simplification. In Proceedings of the 23rd International Conference on Computational Linguistics (Coling 2010), pages 1353-1361. 